\title{
FIELD EXCITED BY PLANE WAVES IN ANISOTROPIC OPTICAL WAVEGUIDE
}

\author{
M. WABIA \\ Institute of Physics, Technical University, Al. Piastów 19, 70-310 Szczecin, Poland
}

(Received June 14, 1991; revised version March 24, 1992)

\begin{abstract}
Displacing a linear source exciting the waveguide along an arbitrary fixed direction to infinity one can create the conditions in which the waveguide is excited by a plane wave. The nature of the excited field in a waveguide is complicated. On the upper plane of the waveguide $x=+l$ one always deals with the radiation mode whereas on the lower boundary of the waveguide $x=-l$ one can deal with the radiation mode or the surface-type wave.
\end{abstract}

PACS numbers: 42.80.Lt

\section{Introduction}

The problem of the excitation of planar optical waveguide has been considered by a number of authors [1-8]. At present, it is practically solved for a case of isotropic optical waveguides.

Thus, an isotropic planar waveguide can be excited by a gaussian beam through its direct focussing on an exposed cross-section $[1,2]$, by a plane wave incident on a waveguide through air gap [3-8] and by a linear source [11].

As it is well-known, in the process of excitation the important role is played by inhomogeneous waves and the effect of optical tunneling. A homogeneous plane wave incident directly on an isotropic waveguide is unable to create, in this structure, inhomogeneous waves which could be transformed further into guided modes. Thus, this wave does not excite a planar isotropic waveguide. In the case of an anisotropic waveguide a physical reality is quite different. Inhomogeneous waves can appear on a boundary between two media both in the refracted and reflected fields $[9,10]$. This is conditioned by more general properties of anisotropic media which cannot be described by a scalar but by a tensor of dielectric permittivity.

This fact suggests a possibility of a further transformation of inhomogeneous waves and a possibility of the construction of such a field like a field guided by a waveguide. As a consequence, it seems possible to excite an anisotropic waveguide directly by a plane wave. 
The reason underlying the present paper is the examination of the nature of electromagnetic waves in anisotropic media and in the situation when boundary conditions are determined in detail. An analysis of the possibility of the uniaxial waveguide excitation by a planar wave is the main aim of the present paper.

We shall seek the solution of the problem using a linear source which placed near a waveguide causes its excitation [12]. Removing this source at a sufficiently large distance (to infinity) we create a situation in which inhomogeneous waves emitted by a source do not reach this structure and a waveguide is excited by a homogeneous plane wave.

\section{Formulation of the problem}

The anisotropic waveguide considered here constitutes a two-dimensional uniaxial structure with three anisotropic layers. The geometry of the system is shown in Fig. 1. The thickness of the film is $2 l$ and the direction of the optic axis

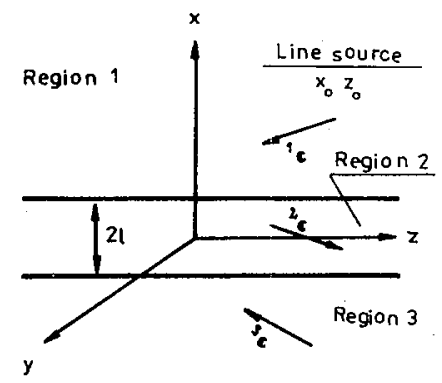

Fig. 1. Geometry of the source-excited uniaxial waveguide. ${ }^{1} c,{ }^{2} c$, and ${ }^{3} c$ are the unit vectors in the directions of the optical axis of layers, respectively .

of each layer is determined by the direction of a unit vector $\boldsymbol{c}$.

The relative permittivity tensors in the $j$-region $(j=1,2,3)$ of the guide corresponding to the optic axis in the $x, z$ plane are all of the form

$$
{ }^{j} \hat{\varepsilon}=\left[\begin{array}{ccc}
{ }^{j} \varepsilon_{11} & 0 & { }^{j} \varepsilon_{13} \\
0 & { }^{j} \varepsilon_{22} & 0 \\
{ }^{j} \varepsilon_{31} & 0 & { }^{j} \varepsilon_{33}
\end{array}\right]
$$

with

$$
\varepsilon_{i j}=\varepsilon^{\circ}\left(\delta_{i j}-c_{i} c_{j}\right)+\varepsilon^{\mathrm{e}} c_{i} c_{j}
$$

where $\varepsilon^{\circ}$ and $\varepsilon^{\mathrm{e}}$ are the ordinary and extraordinary relative permittivities. A harmonically oscillating magnetic linear excitation source is located at $x=x_{0}$, $z=z_{0}$ in the top layer (cover). For simplicity only the TM-type field will be considered here. As it is well-known TM wave propagating along the $z$-direction have a single component of the magnetic field $H_{y}$, from which the electric field components of $E_{x}$ and $E_{z}$ may be found. 
The integral representation of $H_{y}$ has been found for all regions of the waveguide structure. In the regions of interest it has the form [12]

$$
\begin{aligned}
& { }^{1} H_{y}(x, z)=\frac{1}{4 \pi} \int_{-\infty}^{+\infty} \gamma_{1}^{-1} g_{1}(h) \exp \left[-{ }^{1} \varepsilon_{11}^{-1}\left(\gamma_{1}-\mathrm{i}^{1} \varepsilon_{13} h\right) x-\mathrm{i} h z\right] \\
& { }^{2} H_{y}(x, z)=\frac{1}{4 \pi} \int_{-\infty}^{+\infty} \gamma^{-1} g_{2+}(h) \exp \left[-{ }^{2} \varepsilon_{11}^{-1}\left(\gamma_{2}-\mathrm{i}^{2} \varepsilon_{13} h\right) x-\mathrm{i} h z\right] \mathrm{d} h \\
& +\frac{1}{4 \pi} \int_{-\infty}^{+\infty} \gamma_{2}^{-1} g_{2-}(h) \exp \left[{ }^{2} \varepsilon_{11}^{-1}\left(\gamma_{2}+\mathrm{i}^{2} \varepsilon_{13} h\right) x-\mathrm{i} h z\right] \mathrm{d} h \\
& { }^{3} H_{y}(x, z)=\frac{1}{4 \pi} \int_{-\infty}^{+\infty} \gamma_{3}^{-1} g_{3}(h) \exp \left[{ }^{3} \varepsilon_{11}^{-1}\left(\gamma_{3}+\mathrm{i}^{3} \varepsilon_{13} h\right) x-\mathrm{i} h z\right] \mathrm{d} h
\end{aligned}
$$

for $x<-l$, (substrate), where $\gamma_{j}=\left[\alpha_{j}\left(h^{2}-k_{j}^{2}\right)\right]^{1 / 2}, \quad k_{j}=k_{0}\left({ }^{j} \varepsilon_{11}\right)^{1 / 2}, h-$ the propagation constant of the wave in the direction of the $z$-axis.

Spectral densities $g_{1}(h), g_{2}(h), g_{3}(h)$ are given as

$$
\begin{aligned}
& g_{1}(h)=\frac{a+b}{c+d} \exp \left[{ }^{1} \varepsilon_{11}^{-1}\left(\gamma_{1}+\mathrm{i}{ }^{1} \varepsilon_{13} h\right)\left(l-x_{0}\right)\right. \\
& \left.+{ }^{1} \varepsilon_{11}^{-1}\left(\gamma_{1}-\mathrm{i}{ }^{1} \varepsilon_{13} h\right) l+\mathrm{i} h z_{0}\right], \\
& g_{2+}(h)=\frac{q}{c+d} \exp \left[{ }^{1} \varepsilon_{11}^{-1}\left(\gamma_{1}+\mathrm{i}^{1} \varepsilon_{13} h\right)\left(l-x_{0}\right)\right. \\
& \left.-{ }^{2} \varepsilon_{11}^{-1}\left(\gamma_{2}+\mathrm{i}{ }^{2} \varepsilon_{13} h\right) l+\mathrm{i} h z_{0}\right], \\
& g_{2-}(h)=\frac{f}{c+d} \exp \left[{ }^{1} \varepsilon_{11}^{-1}\left(\gamma_{1}+\mathrm{i}{ }^{1} \varepsilon_{13} h\right)\left(l-x_{0}\right)\right. \\
& \left.+{ }^{2} \varepsilon_{11}^{-1}\left(\gamma_{2}-\mathrm{i}{ }^{2} \varepsilon_{13} h\right) l+\mathrm{i} h z_{0}\right], \\
& g_{3}(h)=\frac{m}{c+d} \exp \left[{ }^{1} \varepsilon_{11}^{-1}\left(\gamma_{1}+\mathrm{i}{ }^{1} \varepsilon_{13} h\right)\left(l-x_{0}\right)\right. \\
& \left.-2 \mathrm{i}{ }^{2} \varepsilon_{13}^{-1} h l+{ }^{3} \varepsilon_{11}^{-1}\left(\gamma_{3}+\mathrm{i}{ }^{3} \varepsilon_{13} h\right) l+\mathrm{i} h z_{0}\right],
\end{aligned}
$$

with

$$
\begin{aligned}
& a=\left(\alpha_{2}^{2} \gamma_{1} \gamma_{3}-\alpha_{1} \alpha_{3} \gamma_{2}^{2}\right) \operatorname{sh}\left(2^{2} \varepsilon_{11}^{-1} \gamma_{2} l\right), \\
& b=\alpha_{2} \gamma_{2}\left(\alpha_{3} \gamma_{1}-\alpha_{1} \gamma_{3}\right) \operatorname{ch}\left(2^{2} \varepsilon_{11}^{-1} \gamma_{2} l\right), \\
& c=\left(\alpha_{2}^{2} \gamma_{1} \gamma_{3}+\alpha_{1} \alpha_{3} \gamma_{2}^{2}\right) \operatorname{sh}\left(2^{2} \varepsilon_{11}^{-1} \gamma_{2} l\right), \\
& d=\alpha_{2} \gamma_{2}\left(\alpha_{1} \gamma_{3}+\alpha_{3} \gamma_{1}\right) \operatorname{ch}\left(2^{2} \varepsilon_{11}^{-1} \gamma_{2} l\right), \\
& q=-\alpha_{2} \gamma_{2}\left(\alpha_{2} \gamma_{3}-\alpha_{3} \gamma_{2}\right), \\
& f=\alpha_{2} \gamma_{2}\left(\alpha_{2} \gamma_{3}+\alpha_{3} \gamma_{2}\right), \\
& m=2 \alpha_{2} \alpha_{3} \gamma_{2} \gamma_{3} .
\end{aligned}
$$

The spectral representations (3)-(5) may be interpreted in terms of waves with a continuous spectrum of the eigenfunctions $\exp (-\mathrm{i} h z)$ and real eigenvalues $h$. The direction of propagation is determined here by the direction of the $x$-axis. The shift of a line source to infinity along any constant direction, creates the situation in which the waveguide is excited by a plane wave generated by an infinitely distant source. 


\section{Excitation by plane waves}

The case considered now is equivalent of the displacement to infinity of the actual excitation source. The shift of a line source to infinity along any fixed direction $r \rightarrow \infty, \theta=$ const., creates the conditions justifying the usage of the steepest descent method for the calculation of the excitation fields (3)-(5).

Changing rectangular coordinates $x, z$ for cylindrical ones $r, \theta$ and also changing the variable of integration in the following way:

$$
\begin{aligned}
& \left(x_{0}-l\right)={ }^{1} \varepsilon_{11} r \cos \theta, \\
& z_{0}=-\sqrt{\alpha_{1}} r \sin \theta, \\
& h=k_{1} \sin w,
\end{aligned}
$$

we have

$$
\gamma_{1}=-\mathrm{i} \sqrt{\alpha_{1}} k_{1} \sin w
$$

and

$$
{ }^{1} H_{y}(x, z)=\frac{\mathrm{i}}{4 \pi \sqrt{\alpha}} \int_{-\pi / 2+\mathrm{i} \infty}^{\pi / 2-\mathrm{i} \infty} g_{1}(w) \exp \left[k_{1} r \beta(w)\right] \mathrm{d} w,
$$

where

$$
\begin{aligned}
& g_{1}(w)=\frac{a(w)+b(w)}{c(w)+d(w)} \exp \left[-{ }^{1} \varepsilon_{11}^{-1}\left(\gamma_{1}-\mathrm{i}^{1} \varepsilon_{13} h\right)(x-l)-\mathrm{i} h z\right] \\
& \beta(w)=\mathrm{i}\left(\sqrt{\alpha_{1}} \cos \theta \cos w-\sqrt{\alpha_{1}} \sin \theta \cdot \sin w-{ }^{1} \varepsilon_{13} \cos \theta \sin w\right) .
\end{aligned}
$$

The saddle point $w_{\mathbf{s}}$ is now determined by

$$
\tan w_{\mathrm{s}}=-\left(\tan \theta+\frac{{ }^{1} \varepsilon_{13}}{\sqrt{\alpha_{1}}}\right) \text {. }
$$

The conventional approach for the steepest descent method gives the following result for the magnetic field $H_{y}(x, z)$ in the cover:

$$
\begin{aligned}
& { }^{1} H_{y}(x, z)=\mathrm{i} \alpha_{1}^{-1 / 2}(8 \pi r \eta)^{-1 / 2} \exp \left[\mathrm{i}\left(k_{1} r \eta-\pi / 4\right)\right] \frac{a\left(w_{\mathrm{s}}\right)+b\left(w_{\mathrm{s}}\right)}{c\left(w_{\mathrm{s}}\right)+d\left(w_{\mathrm{s}}\right)} \\
& \times \exp \left[\mathrm{i} k_{1}\left(\alpha_{1} \cos w_{\mathrm{s}}+{ }^{1} \varepsilon_{13} \sin w_{\mathrm{s}}\right)(x-l)+\sin w_{\mathrm{s}} z\right],
\end{aligned}
$$

where

$$
\eta=\left[\alpha_{1} \cos ^{2} \theta+\left(\sqrt{\alpha_{1}} \sin \theta+{ }^{1} \varepsilon_{13} \cos \theta\right)^{2}\right]^{1 / 2} .
$$

The factor $(8 \pi r \eta)^{-1 / 2} \exp \left[\mathrm{i}\left(k_{1} r \eta-\pi / 4\right)\right]=A_{0}(\theta)$ in the expression (25) plays the role of the amplitude for the incident wave (from the $\theta$ direction) emitted by an infinitely distant source.

Thus, (25) can be rewritten as

$$
\begin{aligned}
& { }^{1} H_{y}(x, z)=\mathrm{i} \alpha_{1}^{-1 / 2} A_{0}(\theta) \frac{a\left(w_{\mathrm{s}}\right)+b\left(w_{\mathrm{s}}\right)}{c\left(w_{\mathrm{s}}\right)+d\left(w_{\mathrm{s}}\right)} \\
& \times \exp \left[\mathrm{i} k_{1}\left(\sqrt{\alpha_{1}} \cos w_{\mathrm{s}}+{ }^{1} \varepsilon_{13} \sin w_{\mathrm{s}}\right)(x-l)+\sin w_{\mathrm{s}} z\right] .
\end{aligned}
$$

Based on (27) it is interesting to note that the reflection process taking place is not the mirror one. The reflected wave (27) propagates in the direction 
determined by the $w_{\mathrm{s}}$ angle, while the incident wave arrives from the $\theta$ direction. As seen from (24) only for the case ${ }^{1} \varepsilon_{13}=0$ the reflection is the mirror one. In contrast to the isotropic case we observe here that the amplitude of the incident wave depends on the direction of incidence at the plane interface of the media. In consequence, the amplitude of the reflected wave (25) is evidently a function of the incident angle $\theta$, what is not observed for the analogous isotropic case.

Unfortunately, one must note the fact that the anisotropic waveguide will not be excited by the above-mentioned plane wave. The guiding of the field in the waveguide structure is connected with the capability of supporting the surface wave in this structure, i.e. the wave characterized by an exponential decay away from the waveguide. From (27), (24) and (20) it is easy to verify that such a field which decays exponentially in the cover is not realizable in the case of the excitation by an incident plane wave. The asymptotic expression for the magnetic field in the substrate can also be easily obtained. The resulting approximation is

$$
\begin{aligned}
& { }^{3} H_{y}(x, z)=A_{0}(\theta) m\left(w_{\mathrm{s}}\right)\left[c\left(w_{\mathrm{s}}\right)+d\left(w_{\mathrm{s}}\right)\right]^{-1} \gamma_{3}^{-1}\left(w_{\mathrm{s}}\right) \\
& \times \exp \left[{ }^{3} \varepsilon_{11}^{-1}\left(\gamma_{3}+\mathrm{i}^{3} \varepsilon_{13} k_{1} \sin w_{\mathrm{s}}\right)(x+l)\right. \\
& \left.-2 \mathrm{i}{ }^{2} \varepsilon_{13} k_{1} \sin w_{\mathrm{s}} l-\mathrm{i} k_{1} \sin w_{\mathrm{s}} z\right],
\end{aligned}
$$

where

$$
\gamma_{3}=\left[\alpha_{3}\left(\sin ^{2} w_{s}-k_{3}^{2}\right)\right]^{1 / 2} .
$$

Relations (29) and (28) indicate that along the lower interface in the waveguide the surface-type wave may be supported. This situation exists when

$$
\sin w_{\mathrm{s}}>k_{3} / k_{1} \text {. }
$$

Using (24) and (30), appropriate angle of incidence of the wave generated by an infinitely distant source for the above-described situation may be determined as

$$
\tan \theta>k_{3}\left(k_{1}^{2}-k_{3}^{2}\right)^{-1 / 2}-{ }^{1} \varepsilon_{13} \alpha_{1}^{-1 / 2} .
$$

In the case of $k_{3}>k_{1}$, the lower interface in the waveguide cannot support the surface-type wave for the angle of incidence $\theta$.

For the electromagnetic field excited in the film by an infinitely distant line source, the steepest descent method may also be applicable and we obtain for this case

$$
\begin{aligned}
& { }^{2} H_{y}(x, z)=-\mathrm{i} A_{0}(\theta) \gamma^{\prime-1} \frac{q\left(w_{\mathrm{s}}\right)}{c\left(w_{\mathrm{s}}\right)+d\left(w_{\mathrm{s}}\right)} \\
& \times \exp \left[\mathrm{i}^{2} \varepsilon_{11}^{-1}{\gamma^{\prime}}_{2}(l+x)-\mathrm{i}^{2} \varepsilon_{11}^{-1}{ }^{2} \varepsilon_{13} k_{1} \sin w_{\mathrm{s}}(l-x)-\mathrm{i} k_{1} \sin w_{\mathrm{s}} z\right] \\
& -\mathrm{i} A_{0}(\theta){\gamma^{\prime}}_{2}^{-1} \frac{f\left(w_{\mathrm{s}}\right)}{c\left(w_{\mathrm{s}}\right)+d\left(w_{\mathrm{s}}\right)} \\
& \times \exp \left[-\mathrm{i}{ }^{2} \varepsilon_{11}^{-1} \gamma_{2}^{\prime}(l+x)-\mathrm{i}^{2} \varepsilon_{11}^{-1}{ }^{2} \varepsilon_{13}^{-1} k_{1} \sin w_{\mathrm{s}}(l-x)-\mathrm{i} k_{1} \sin w_{\mathrm{s}} z\right],
\end{aligned}
$$

where

$$
\gamma_{2}^{\prime}=\left[\alpha_{2}\left(k_{2}^{2}-k_{1}^{2} \sin ^{2} w_{\mathrm{s}}\right)\right]^{1 / 2} .
$$

Since $k_{2}>k_{1}$, it is seen from (33) that $\gamma_{2}^{\prime}$ is real. Therefore, the field described by (32) is a propagating mode. 
The nature of the field in the cover and the substrate as well as the continuity conditions indicate for a complicated character of the wave motion phenomenon in the film. On the upper interface $x=+l$ this is always the radiation mode, on the lower interface $x=-l$ this is the mode of radiation only when $k_{3}>k_{1}$ or alternatively this is the surface-type wave when $k_{3}<k_{1}$.

Taking into account that the investigated field is far from the source we can easily deduce from (27), (28) and (32) that the surface-type wave shows all the properties of the lateral wave, i.e. propagates along the interface, decays exponentially in the direction perpendicular to the interface in the substrate and is radiated from the interface into the film. This wave is in fact the lateral wave.

\section{Conclusion}

The shift of a line source to infinity along any constant direction $r \rightarrow \infty, \theta=$ const., creates the situation in which the waveguide is excitable by the plane waves generated by an infinitely distant source. Unfortunately, one must note the fact that the anisotropic waveguide will not be excited by the above-mentioned waves in the sense of guiding of the surface waves by both interfaces. In this case the nature of the electromagnetic wave propagation in the waveguide is complicated. On the upper interface $x=+l$ this is always the radiation mode; on the lower interface $x=-l$ this is the mode of radiation only when $k_{3}>k_{1}$ or alternatively it is the lateral wave when $k_{3}<k_{1}$. The anisotropy of the media also essentially influences on the character of electromagnetic field propagation. The reflection and the total internal reflection are the processes which are not symmetrical (not mirror) ones. The amplitudes of the field depend strongly on the direction of propagation.

The obtained results have rather obvious physical sense. As it is well-known $[11,12]$ inhomogeneous waves are responsible for the excitation of surface modes in waveguide structures as well as flat interfaces. Their decissive role played in the excitation process of waveguides is presented in this paper.

The spectrum of waves emitted by a linear source contains, among others, also inhomogeneous waves. In the case when this source is placed not far from the layer (region 2), a uniaxial waveguide can be excited by such a source, i.e. can guide surface waves.

With the increase of the distance up to infinity we deal with the situation when the emitted inhomogeneous waves do not reach the layer (region 2) because they vanish rapidly. Then, only the homogeneous plane waves penetrate the layer without exciting surface modes.

An interesting case of guidance of lateral waves by the lower layer $x=-l$ can take place without the contribution from inhomogeneous waves. This occurs when $k_{1}>k_{3}$ and the homogeneous wave incides from the direction of (31). This wave undergoes then the total internal reflection from the lower layer and partial reflection from the upper layer resulting into a pseudosurface wave (lateral wave) guided by the boundary $x=-l$, i.e. the wave vanishing exponentially in the region 3 and oscillating in the region 2. 


\section{Acknowledgments}

The author expresses his gratitude to Prof. J. Petykiewicz and to Dr. K. Gniadek for many helpful discussions connected with this research.

\section{References}

[1] R. Shubert, J.H. Harris, IEEE Trans. Microwave Theory Tech. MTT-16, 1048 (1968).

[2] D. Marcuse, E.A.J. Marcatili, Bell System Techn. J. 50, 43 (1971).

[3] J.H. Harris, R. Shubert, J.N. Polky, J. Opt. Soc. Am. 60, 1007 (1970).

[4] P.K. Tien, R. Ulrich, J. Opt. Soc. Am. 60, 1325 (1970).

[5] R. Urlich, J. Opt. Soc. Am. 60, 1337 (1970).

[6] J.E. Midwinter, J. Quant. Electron. QE-7, 345 (1971).

[7] T. Tamir, L.H. Bertoni, J. Opt. Soc. Am. 61, 1397 (1971).

[8] R. Ulrich, J. Opt. Soc. Am. 61, 1467 (1971).

[9] M. Wabia, Acta Phys. Pol. A78, 861 (1990).

[10] M. Wabia, Acta Phys. Pol. A78, 873 (1990).

[11] T. Tamir (Ed.), Integrated Optics, Springer-Verlag, Berlin, Heidelberg, New York 1975.

[12] M. Wabia, K. Gniadek, Acta Phys. Pol. A54, 493 (1978). 